\title{
The Hybrid Traffic Offloading Mode for Disaster-Resilient Communication Networks Based on User Mobility
}

\author{
Ang-Hsun Tsai $\mathbb{D}^{1}$ and Chung-Hsien Tsai $\mathbb{D}^{2}$ \\ ${ }^{1}$ National Yang Ming Chiao Tung University, Taiwan \\ ${ }^{2}$ Chung Cheng Institute of Technology, National Defense University, Taiwan \\ Correspondence should be addressed to Ang-Hsun Tsai; anghsun@gmail.com
}

Received 8 July 2021; Revised 21 August 2021; Accepted 30 August 2021; Published 20 October 2021

Academic Editor: Chao-Yang Lee

Copyright (C) 2021 Ang-Hsun Tsai and Chung-Hsien Tsai. This is an open access article distributed under the Creative Commons Attribution License, which permits unrestricted use, distribution, and reproduction in any medium, provided the original work is properly cited.

\begin{abstract}
Emergency communication systems play a major role in disaster-relief environments. In terms of the public safety research, the emergency relief communication system can provide a high system capacity for networks based on the development of LongTerm Evolution. However, in the event of a disaster, mass traffic information can cause congestion in the core network, and communications between relief workers may be limited. Consequently, spectrum efficiency can be very weak. This paper provides a hybrid traffic offloading mechanism combining Device-to-Device (D2D) and Local IP Access (LIPA) modes for the disaster-resilient network. With receiving power, the distance between relief workers and the distance between relief workers and the vehicular eNodeB (VeNB) as the LIPA/D2D switching criteria, the network can select an appropriate mode to prevent core network congestion. This paper also considers the effects of the mobility models (i.e., random walk and random direction) on the spectrum efficiency of the disaster-resilient communication system. The proposed hybrid LIPA/D2D traffic offloading mechanism can prevent the local communication traffic from flowing into the core network and significantly improve the system spectrum efficiency when the core network is under congestion. Therefore, the proposed mechanism can effectively improve the quality of the communication between relief workers served by the same VeNB for performing rescue operations. Moreover, the hybrid LIPA/D2D traffic offloading mechanism can be applied to the smart city and smart home in the future.
\end{abstract}

\section{Introduction}

1.1. Background and Motivation. The evolution of mobile communication systems from the first generation $(1 G)$ to the third generation $(3 \mathrm{G})$ is still essentially based on the philosophy of conventional telecommunication systems. These mobile communication systems are stable and reliable for voice services but lack sufficient bandwidth and operational resilience for multimedia data services. These deficiencies are well corrected in the fourth generation $(4 \mathrm{G})$ mobile communication system. The $4 \mathrm{G}$ mobile communication system effectively enhances system capabilities and resolves channel effects and interference to bring a new high-speed Internet experience to users. In addition to the improved network architecture, the $4 \mathrm{G}$ mobile communication system is equipped with advanced signal processing technology that enhances the effectiveness of the receiver. In the framework of the IP-based $4 \mathrm{G}$ communi- cation network architecture [1], when a user equipment (UE) communicates with an adjacent receiver within an evolved node B (eNodeB or eNB) service area, data packets shall be transmitted through a radio access network (RAN) to the evolved packet core (EPC) and should then be transferred to the receiver along the original path. The disadvantage of such a transmission link is that all data needs to be transmitted by many network devices and Internet service providers (ISPs) during transmission. In this case, an increase in transmission traffic may lead to high latency and congestion of the core network.

With the dramatic global climate change, different types of natural disasters occur more frequently, resulting in serious social and economic losses and even endangering the lives of the general population. However, where there are major natural disasters (e.g., windstorms, floods, earthquakes, tsunamis, and landslides), buildings, roads, and infrastructure related 
to the livelihoods of the general public may be destroyed, complicating rescue operations. In the Great East Japan Earthquake, for example, most eNBs were destroyed, and only a few eNBs could normally provide communication services. Thirty minutes after the earthquake, the total number of calls increased by a factor of eight [2]. The significant increase in data packets is due to the safety confirmation of the general public and their families, instant media reports on the network media, and messages during search and rescue (SAR) operations. Within a short time, a large amount of communication traffic was generated and transmitted to the core network, causing congestion of the core network. Consequently, the delay of the packets or even the abandonment took place, reducing the efficiency of the spectrum in the disaster area.

For a disaster-resilient communication network in the disaster area, an efficient traffic offloading mechanism is necessary. With a rescue operation, the relief workers in the disaster area may randomly move to places where there may be people in help. The relief workers may move by walking or by car, and the mobilities of the relief workers are different. The distance between relief workers are changing all the time. Some messages are passed between workers, and some messages need to be reported back to the command center. Not all the traffic needs to go through the core network. The disaster-resilient communication network needs the traffic offloading mechanism to differentiate between the internal messages and the external messages. In addition, the traffic offloading mechanism should avoid a large amount of disaster relief communication traffic passing through the core network to result in the congestion problem.

This paper considers the use of two traffic offloading mechanisms [3] to resolve the congestion problem faced by the emergency communication network. Using the traffic offloading technology, the communication traffic generated in the disaster area can be transmitted directly or indirectly to the receiver, thereby reducing the communication traffic back to the core network. This can effectively prevent core network congestion from causing a low spectrum efficiency in the disaster area and thereby prevent the interruption of internal communication in the disaster area. One mechanism is Local IP Access (LIPA) [4], in which UE data is directly distributed (or accessed) from eNBs without being transmitted to the core networks of ISPs, as shown in Figure 1. In the case of data transmission between UEs and other nodes in a near end network, local packets can be directly routed via the eNB to the receiver (UE), without being transmitted to the core network. In this way, the transmission delay can be reduced, the traffic can be offloaded, and the load of the core network and the transmission costs can be reduced. The other mechanism is the direct Deviceto-Device (D2D) communication [5], in which the devices communicate directly with each other, without being routed through the eNB. In D2D mode $[6,7]$, spectral resources of the network can be utilized repeatedly to enhance the spectrum efficiency and reduce the load of eNB. The D2D mode is used for communication between adjacent devices. It enables direct communication between devices in order to reduce the backhaul traffic and thereby achieve the offloading effect of traffic.
1.2. Related Works. In recent years, owing to the requirements to improve the system data traffic and reduce the core network load, interest in D2D communication technology has increased. To respond to the demand for more data and less delay in next-generation communication systems, D2D is applied in the existing cellular system, which has become the trend of future communication systems $[8,9]$. However, most studies have not examined the potential effects of UE mobility models in actual situations on the transmission rate of communication in the disaster area.

Some studies did not consider the mobility of users in the disaster-resilient communication network, as shown in Table 1. In actual situations, relief workers have different mobility models depending on their operational needs. For example, when relief workers are informed of the exact location of a victim, they perform an SAR operation within the key area only; when relief workers perform casualty transport or fixed-point movement operation in the disaster area, they move at a fixed rate and in a fixed direction. However, in the studies of References [8, 10-14], the simulation scenarios do not account for the mobility of the relief workers in the disaster area. To make the simulation scenario better accord with the actual situation, this paper considered the possible impacts of UE mobility on the communication traffic offloading.

Some papers considered the mobility of users for the D2D communication network, such as $[15,16]$ and [17, $18]$, as shown in Table 1 . The authors in $[15,16]$ designed a suitable group-based data synchronization method and proposed a mobility-aware data update mechanism to improve the overhead of maintaining the consistency of shared data items in an MP2P network. A theoretical analysis of transmission rate on $\mathrm{D} 2 \mathrm{D}$ mode selection with user mobility has been investigated in [18]. However, the papers [15-18] only considered the D2D mode and did not consider the other traffic offloading mechanism.

1.3. Objective. This paper proposes a hybrid LIPA/D2D traffic offloading mechanism for a disaster-resilient communication network. When a disaster occurs that results in a lack of communication resources in the disaster area, a vehicular eNodeB (VeNB) enters the disaster area, and each relief worker is equipped with a UE that can instantly report his or her global positioning system (GPS) location coordinates. In this case, the hybrid mechanism can switch to a better mode of traffic offloading from D2D or LIPA depending on the distance and received power between the VeNB and relief workers. In addition, this paper investigates the impact of different mobility models such as random walk and random direction during rescue operations on the overall transmission rate, in order to evaluate the efficiency of the hybrid LIPA/D2D traffic offloading mechanism.

1.4. Our Contributions. Under the disaster-resilient communication network proposed in this paper, the hybrid LIPA/D2D traffic offloading mechanism can switch the suitable traffic offloading mode when the core network is congested. Therefore, the hybrid LIPA/D2D traffic offloading mechanism can avoid a large amount of disaster relief communication traffic passing 


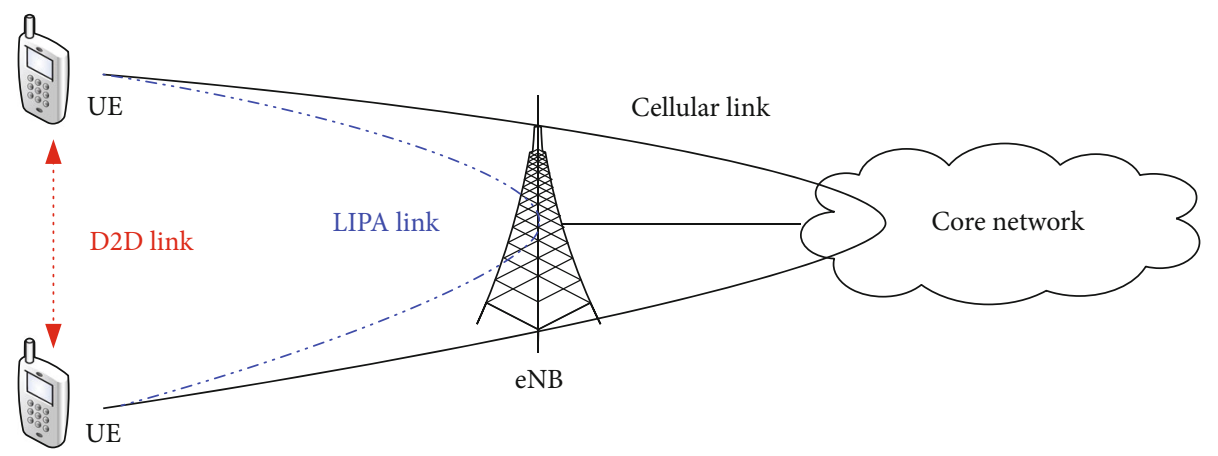

FIGURE 1: Schematic of the communication model.

TABLE 1: Application of synthesis between LIPA/D2D and cellular network.

\begin{tabular}{lccc}
\hline Related works & Method & Mobility & Criterion \\
\hline Doppler et al. [8] & D2D & - & Throughput \\
Frlan [11] & D2D & - & Received power \\
Tang and Ding [14] & D2D & - & Local IP \\
Sankaran [13] & LIPA & - & - \\
Khan et al. [12] & LIPA & - & - \\
Chang et al. [10] & LIPA & - & - \\
Orsino et al. [17] & D2D & Random walk \\
Lai et al. [15, 16] & D2D & Random way point & Time \\
Xu et al. [18] & D2D & Random way point & Received power \\
Our work & Hybrid LIPA/D2D & Random walk; random direction & Received power; distance \\
\hline
\end{tabular}

through the core network and causing the delay or interruption of the internal communication network in the disaster area. In addition, the mobilities of the relief workers may be different according to the disaster environment. The single traffic offloading mechanism cannot support the rescue operation. The hybrid LIPA/D2D traffic offloading mechanism can provide the proper link for the relief workers for improving the spectrum efficiency. The simulation results show that the hybrid D2D and LIPA traffic offloading mechanism can further improve the spectrum efficiency of the disaster-resilient communication system under the core network congestion. Therefore, the operation of the disaster-resilient communication network can be maintained, and the transmission of important information can be achieved in the disaster area.

The hybrid LIPA/D2D traffic offloading mechanism also can be applied to other scenes, such as smart city and smart home. When in a crowded area, the traffic for some users may be within the area. At this time, the network can first distinguish the traffic within the area or the traffic that needs to be sent to the core network. If the traffic belongs to the same coverage of the eNB, our traffic offloading mechanism can provide the location-based services (LBS) based on the users' locations. When the two users are close enough, the network can switch to the D2D mode for the users, while the LIPA mode can be switched when the two users are too far apart. This is a very useful mechanism for smart city and smart home. In the future, if the traffic can be actually measured, the traffic offloading model will be trained by the machine learning and other artificial intelligence techniques for satisfying the demands of various smart cities and smart homes.

The remainder of this paper is organized as follows. Section 2 introduces a disaster relief scenario, channel model, mobility models, and performance metrics. The proposed hybrid LIPA/D2D mode is detailed in Section 3. Section 4 shows the simulation results, and the concluding remarks are given in Section 5.

\section{System Model}

2.1. System Architecture. A disaster-resilient communication network is considered as shown in Figure 2. In the event of a disaster, the terrestrial communication infrastructure in the disaster area may be destroyed, and the communication services may be interrupted or unstable. The governmental organizations (e.g., Executive Yuan in Taiwan) set up the Central Emergency Operation Center (CEOC) to support the rescue operations. At this time, a disaster relief team and a vehicular eNodeB (VeNB) enter the disaster area to establish a Forward Command Post (FCP) and set up an emergency communication network as soon as possible so that the relief workers in the disaster area can perform the rescue, communication/coordination, and disaster information notification. Relief workers can be regarded as mobile user equipments (MUEs) because they must constantly perform SAR and provide disaster information. The FCP 


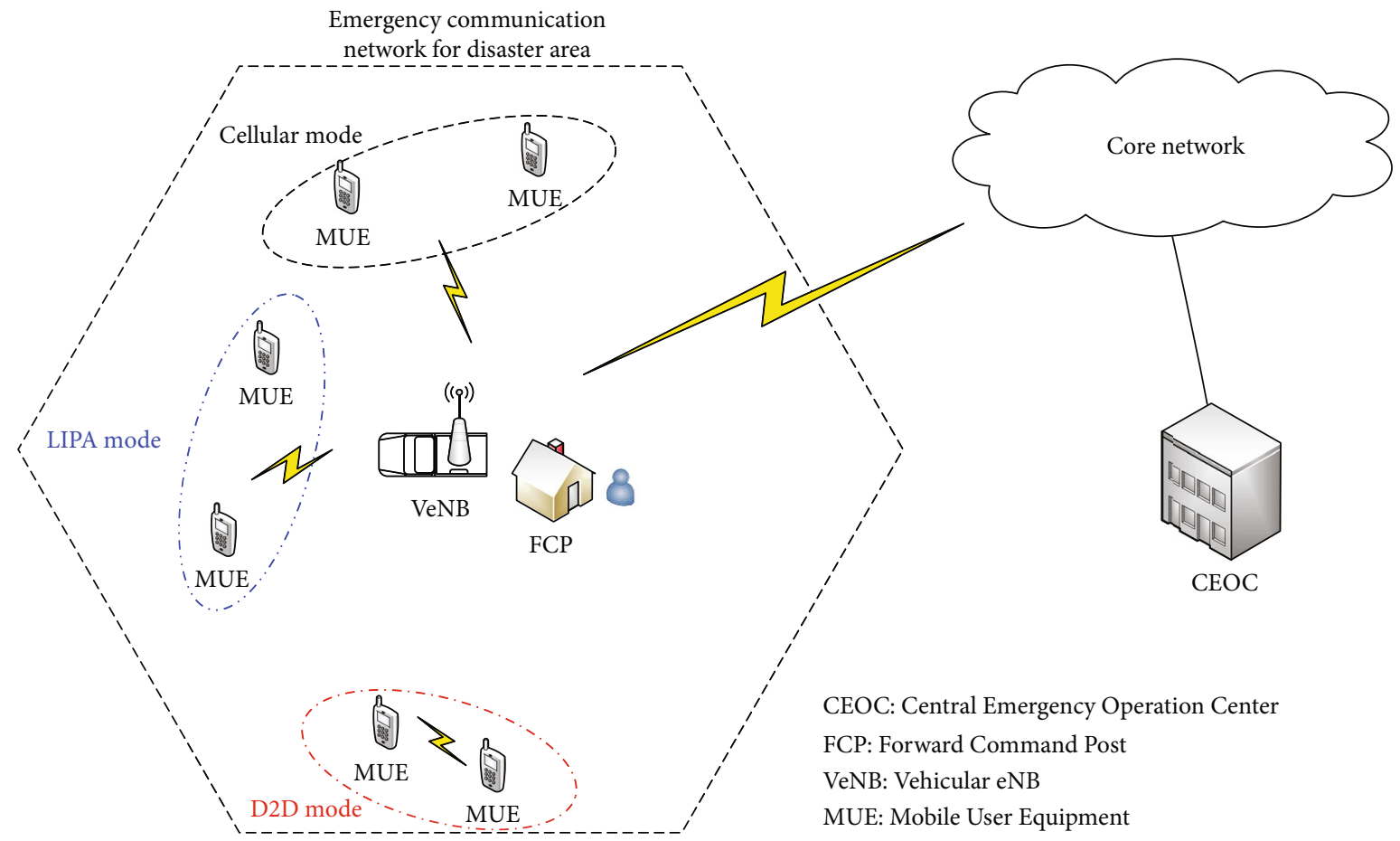

FIgURe 2: A disaster-resilient communication network.

commander will collect all types of disaster information to develop a common operation picture (COP) and report to the CEOC in real time, so that the CEOC can effectively manage the overall situation of the disaster area. In view of the fact that the wired communication infrastructure has been destroyed, the emergency communication network in this context connects to the core network through the wireless transmission.

As shown in Figure 1, when relief workers perform communication/coordination and disaster information reporting in rescue operations, there are mainly three communication modes: cellular mode, Local IP Access (LIPA) mode, and Device-to-Device (D2D) mode. In the cellular mode, the information for intra-area communication/coordination between two devices must be transmitted to the eNodeB (eNB) and the core network before being sent back to the destination device. In the LIPA mode, the information for intra-area communication/coordination between two devices can be transmitted by the eNB directly to the destination device, without being transmitted to the core network. In the D2D mode, the information for intra-area communication/coordination between two devices can be transmitted directly, without being routed via the eNB.

2.2. Channel Model. This paper considers the large-scale fading phenomenon, including two components: (1) path loss caused by the distance between the transmitter and receiver and (2) shadowing caused by the obstacles in the channel. In the simulation scenario of this study, different path losses are set for the LIPA and D2D modes according to References [19-24], as shown in Equation (1). There must be path losses, regardless of the distance between the devices. According to the technical reports of the Third-Generation Partnership Project (3GPP), the path loss can be calculated as a function of the interdevice distance (in $\mathrm{km}$ ) and can be expressed as

$$
L(d)_{d B}=\left\{\begin{array}{l}
128.1+37.6 \log _{10}\left(d_{\text {cellular }}\right) \\
148+40 \log _{10}\left(d_{\mathrm{D} 2 \mathrm{D}}\right)
\end{array},\right.
$$

where $d_{\text {cellular }}$ is the distance from the VeNB to the device and $d_{\mathrm{D} 2 \mathrm{D}}$ is the distance between one pair of devices. Generally, the shadowing effect is represented by a log-normal random variable $(\xi)$, which ranges from $6 \mathrm{~dB}$ to $10 \mathrm{~dB}$. Because the disaster area selected in this paper is subject to the shadowing effect of the complex terrain, the shadowing effect in LIPA and D2D modes is set to the maximum value (i.e., $10 \mathrm{~dB}$ ) [19]. In addition to the large-scale fading phenomenon, this paper also considers the small-scale fading phenomenon in the channel model. The small-scale fading is considered as the Rayleigh fading channel.

2.3. Mobility Module. When relief workers are performing search and rescue (SAR) operations in the disaster area, they must adapt their mobility models to different terrain constraints or operational needs due to the complex terrain of the disaster area. Therefore, in this paper, a mobility module has been designed based on the mobility characteristics of relief workers to simulate the mobility patterns during their SAR operations in the disaster area. This module supports two mobility models: random walk model (RWM) and random direction model (RDM).

The RWM can be used to establish a path along for a relief worker to move at a fixed rate in a random direction. 
In the beginning, the RWM randomly generates an azimuth angle from $[0,2 \pi]$ as the initial moving direction for a relief worker. The relief worker moves to the next search location at a fixed rate. Then, the RWM randomly regenerates an azimuth angle again from $[0,2 \pi]$ as the next moving direction for the relief worker. This paper assumes that the RWM updates the azimuth angle every second. The RWM can simulate a scenario where a relief worker may keep changing the moving direction when the relief worker is unsure of the key SAR area.

The RDM can be used to simulate that the relief worker has to change a path owing to the constraint of communication coverage. Similar to the RWM, the RDM randomly selects an azimuth angle from $[0,2 \pi]$ as the initial moving direction for a relief worker at first. However, the relief worker keeps moving to the boundary of the communication coverage at a fixed rate. Then, the RDM randomly regenerates an azimuth angle of reflection within the communication coverage from $[0,2 \pi]$ as the following moving direction for the relief worker. Different from the RWM, the RDM updates the azimuth angle of reflection whenever the relief worker moves to the boundary of the communication coverage. The RDM can simulate a scenario in which a relief worker needs to return from the edge of the disaster area.

Figure 3 illustrates the results of the mobility module (i.e., RWM and RDM) simulating two relief workers moving to perform the SAR operation in the disaster area at velocities of $3 \mathrm{~km} / \mathrm{h}$ in Figure $3(\mathrm{a}), 10 \mathrm{~km} / \mathrm{h}$ in Figure $3(\mathrm{~b})$, and $30 \mathrm{~km} / \mathrm{h}$ in Figure 3(c), respectively. The simulation scenario is that the VeNB (marked with a diamond) enters the disaster area after the occurrence of the disaster. The area inside the hexagon represents the service area of the VeNB, and two relief workers are combined into a team to enter the disaster area for SAR. The distance between the initial locations of the two relief workers is set as 10 meters, and they constantly move to perform SAR operations within one hour. The movement of the relief workers has been simulated at different speeds of $3 \mathrm{~km} / \mathrm{h}, 10 \mathrm{~km} / \mathrm{h}$, and $30 \mathrm{~km} / \mathrm{h}$, whether by walking or by a vehicle.

2.4. Efficiency Indicators. The signal-to-noise ratio (SNR) is commonly used to evaluate the performance of systems. As shown in Equation (2), if the signal power is large, then the system efficiency and transmission quality will be high. The SNR has a direct effect on the bit error rate and also influences the information capacity. The ratio of the signal power $(S)$ to the noise power $\left(N_{0}\right)$ is shown as

$$
\mathrm{SNR}=\frac{S}{N_{0}} .
$$

For channel characteristics, the large-scale fading (i.e., path loss and shadowing effect) and small-scale fading (i.e., multipath fading) are considered. In this paper, we consider only one VeNB and the UEs of two relief workers in the disaster area. For the LIPA mode, the VeNB has no interference in the uplink, and the UEs have no interference in the downlink. Therefore, the signal-to-noise ratio (SNR) of the VeNB in the uplink and that of the UE in the downlink can be expressed, respectively, as

$$
\begin{gathered}
\gamma_{t}^{\mathrm{UL}}=\frac{P_{a} \cdot 10\left(\xi_{\mathrm{UL}, t} / 10\right) \cdot\left|h_{\mathrm{UL}, t}\right|^{2} / 10^{\left(L\left(d_{\mathrm{UL}, t}\right) / 10\right)}}{N_{0}}, \\
\gamma_{t}^{\mathrm{DL}}=\frac{P_{b} \cdot 10\left(\xi_{\mathrm{DL}, t} / 10\right) \cdot\left|h_{\mathrm{DL}, t}\right|^{2} / 10^{\left(L\left(d_{\mathrm{DL}, t}\right) / 10\right)}}{N_{0}},
\end{gathered}
$$

where $P_{a}$ and $P_{b}$, respectively, represents the transmit power of a UE and the VeNB, and both of their units are watts. $t$ represents the time duration of SAR of relief workers, i.e., the total simulation time. $N_{0}$ represents the noise power. $d_{\mathrm{UL}}$ represents the distance between a relief worker and the VeNB in the uplink, and $d_{\mathrm{DL}}$ represents the distance between the VeNB and a relief worker in the downlink. $L$ represents the path loss calculated according to Equation (1). $\xi_{\mathrm{UL}, t}$ and $\xi_{\mathrm{DL}, t}$ are a log-normal random variable to calculate the shadowing effect in the uplink and downlink, respectively. $h_{\mathrm{UL}, t}$ and $h_{\mathrm{DL}, t}$ represent the Rayleigh fading channel in the uplink and downlink, respectively.

Similarly, there is no interference in the D2D mode, and we assume that the D2D mode is only available in the uplink phase. Therefore, the SNR for D2D communication can be expressed as

$$
\gamma_{t}^{\mathrm{D} 2 \mathrm{D}}=\frac{P_{a} \cdot 10\left(\xi_{D, t} / 10\right) \cdot\left|h_{D, t}\right|^{2} / 10^{\left(L\left(d_{D, t}\right) / 10\right)}}{N_{0}},
$$

where $d_{D, t}$ represents the distance between the devices, $\xi_{D, t}$ is a log-normal random variable to calculate the shadowing effect, and $h_{D, t}$ represents the Rayleigh fading channel.

According to Equations (3) to (5), the theoretical spectrum efficiency of LIPA, D2D, and hybrid LIPA/D2D modes within the time $T$ is expressed as

$$
\begin{gathered}
\mathrm{SE}_{\mathrm{LIPA}}=\frac{1}{T} \sum_{t=1}^{T} \log _{2}\left(1+\min \left\{\gamma_{t}^{\mathrm{UL}}, \gamma_{t}^{\mathrm{DL}}\right\}\right), \\
\mathrm{SE}_{\mathrm{D} 2 \mathrm{D}}=\frac{1}{T} \sum_{t=1}^{T} \log _{2}\left(1+\gamma_{t}^{\mathrm{D} 2 \mathrm{D}}\right), \\
\mathrm{SE}_{\mathrm{D} 2 \mathrm{D}}=\frac{1}{T} \sum_{t=1}^{T} \log _{2}\left(1+\max \left\{\min \left\{\gamma_{t}^{\mathrm{UL}}, \gamma_{t}^{\mathrm{DL}}\right\}, \gamma_{t}^{\mathrm{D} 2 \mathrm{D}}\right\}\right) .
\end{gathered}
$$

In Equations (6) to (8), $\mathrm{SE}_{\mathrm{LIPA}}, \mathrm{SE}_{\mathrm{D} 2 \mathrm{D}}$, and $\mathrm{SE}_{\mathrm{Hybrid}}$ represent the theoretical spectrum efficiency in the LIPA, D2D, and hybrid LIPA/D2D modes, respectively. $t$ represents the start time of SAR, and $T$ represents the end time of SAR. In the LIPA mode, assuming that the timeslots are divided into uplink and downlink timeslots, and the total transmission rate is limited by the minimum transmission rate of the uplink and downlink. Therefore, the average effective spectrum efficiency is the lower one between the uplink SNR and downlink SNR. However, the theoretical spectrum efficiency in the D2D mode is calculated directly using the SNR of the D2D link. In the hybrid LIPA/D2D mode, the higher one between the SNR (LIPA) and SNR (D2D) in each 

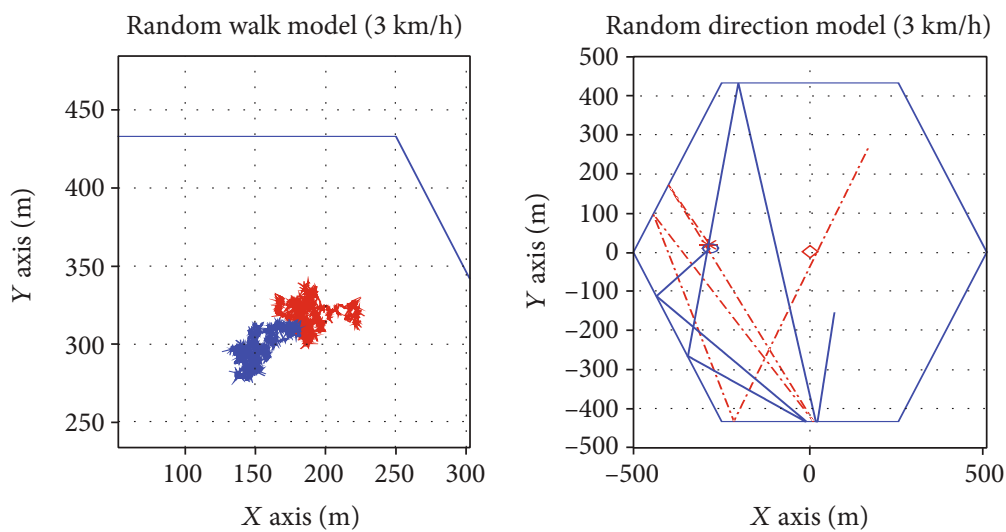

(a) The mobility module of $3 \mathrm{~km} / \mathrm{h}$
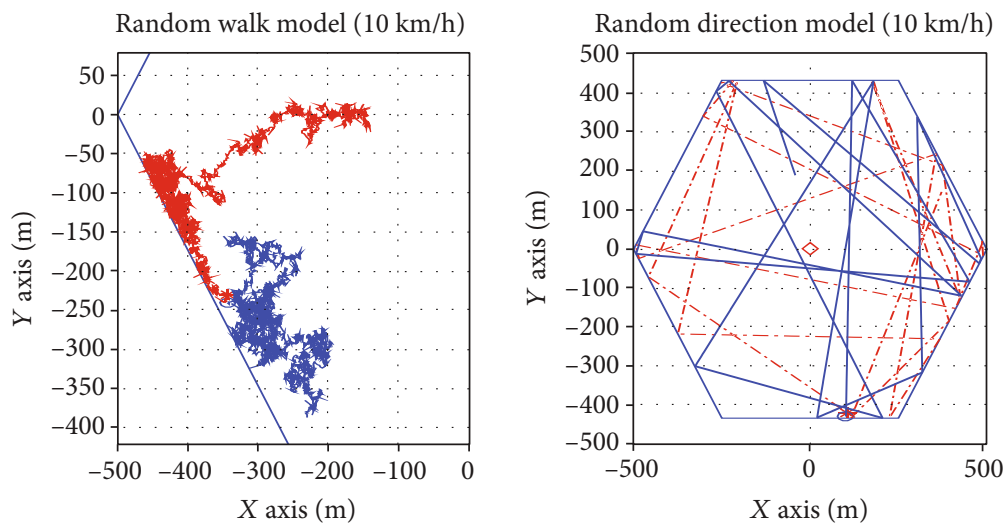

(b) The mobility module of $10 \mathrm{~km} / \mathrm{h}$
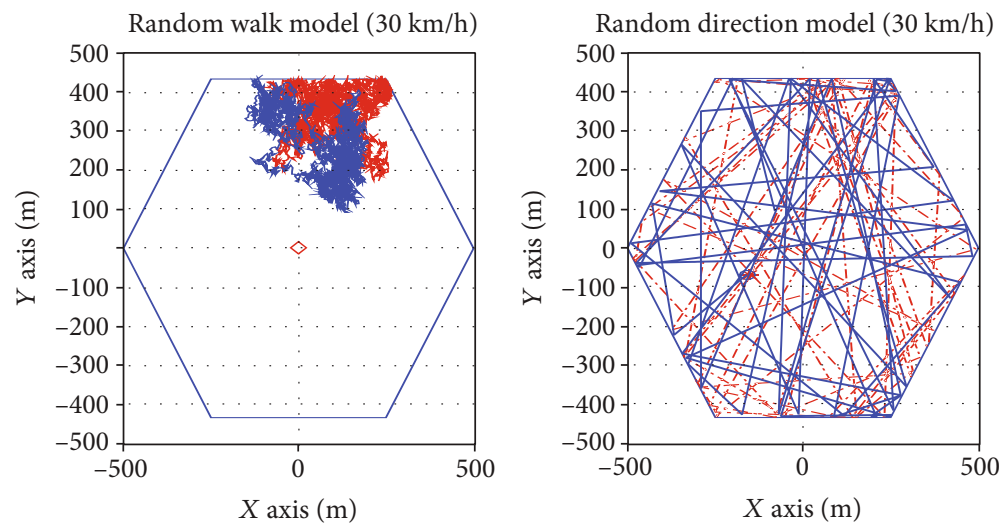

(c) The mobility module of $30 \mathrm{~km} / \mathrm{h}$

Figure 3: Simulation trajectory of the mobility module.

timeslot is used as the theoretical spectrum efficiency of the hybrid LIPA/D2D traffic offloading mechanism.

In this paper, we consider the modulation and coding schemes (MCSs) to mapping the effective spectrum efficiency instead of Shannon's Law in the LTE communication system. That is because the spectrum efficiency calculated by Shannon's Law is the theoretical value. The MCSs have three modulation modes, namely, QPSK, 16-QAM, and 64-QAM with different code rates. Table 2 lists the considered MCSs, the corresponding received SNR, and the effective spectrum efficiency $\Gamma$. With the information of received SNR, the MCS and the corresponding effective spectrum efficiency can be determined according to Table 2. This paper uses the average effective spectrum efficiency as an indicator for performance evaluation.

\section{Hybrid LIPA/D2D Mode}

As shown in Figure 4, in normal cases, the cellular connection is performed between UEs through the general cellular communication architecture. When a disaster occurs, a VeNB enters the disaster area, and an external communication 
TABLE 2: Modulation and coding schemes.

\begin{tabular}{lccc}
\hline $\begin{array}{l}\text { Modulation } \\
\text { Scheme }\end{array}$ & $\begin{array}{c}\text { Code } \\
\text { Rate }\end{array}$ & $\begin{array}{c}\text { Received } \\
\text { SNR }(\mathrm{dB})\end{array}$ & $\begin{array}{c}\text { Effective spectrum } \\
\text { Efficiency } \Gamma(\mathrm{b} / \mathrm{s} / \mathrm{Hz})\end{array}$ \\
\hline QPSK & $1 / 2$ & -2.5 & 0.25 \\
QPSK & $1 / 2$ & 0.5 & 0.5 \\
QPSK & $1 / 2$ & 3.5 & 1 \\
QPSK & $3 / 4$ & 6.5 & 1.5 \\
16-QAM & $1 / 2$ & 9 & 2 \\
16-QAM & $3 / 4$ & 12.5 & 3 \\
64-QAM & $1 / 2$ & 14.5 & 3 \\
64-QAM & $2 / 3$ & 16.5 & 4 \\
64-QAM & $3 / 4$ & 18.5 & 4.5 \\
\hline
\end{tabular}
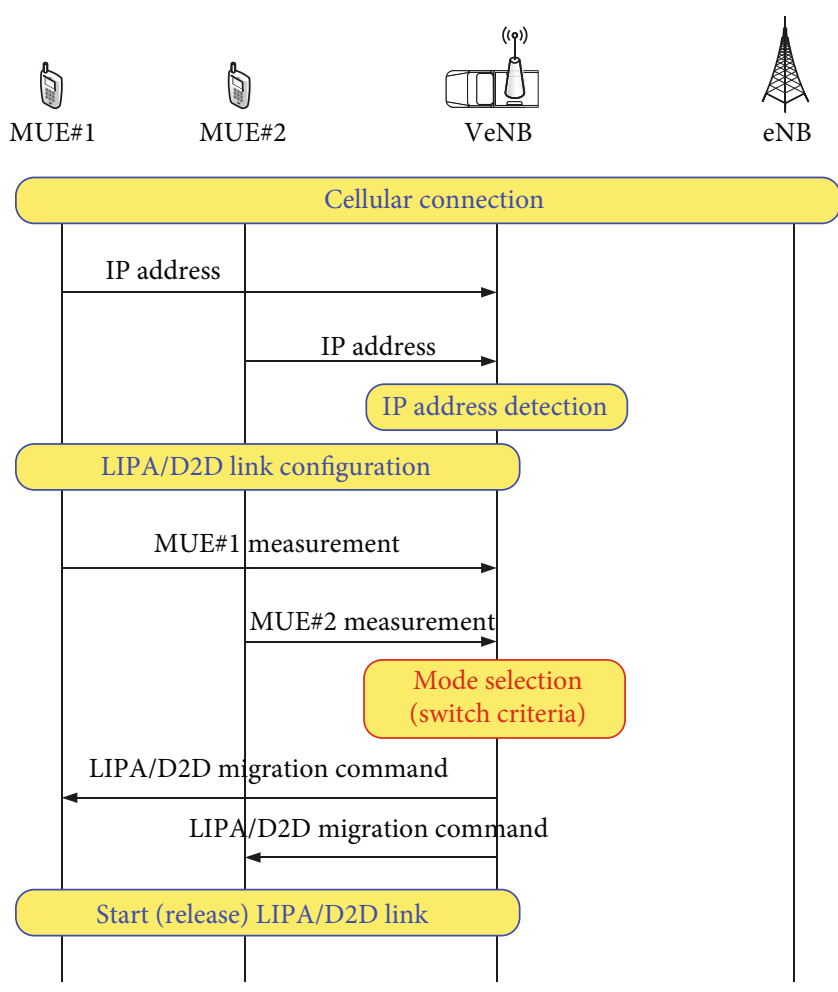

FIGURE 4: A flowchart of the hybrid LIPA/D2D traffic offloading mechanism in the cellular communication mode.

network is constructed. Through the local IP address detection, the VeNB can take over from the destroyed eNB to provide communication services. Since the communication and coordination of relief workers occur primarily within the inside of the disaster area, this paper mainly investigates the switching of intra-area communication modes. After taking over the communication services, the VeNB can measure the reference signal intensity and the GPS location information of MUEs. Then, the VeNB rapidly evaluates the LIPA/D2D downlink transmission in every time slot and notifies the MUEs of the mode switching to obtain the maximum average spectrum efficiency.
The distance and the received power are typically used as criteria for mode switching to investigate the differences in the spectrum efficiency, as shown in Figure 5. After the VeNB enters the disaster area and an external communication network is built, the distance between relief workers $\left(d_{3}\right)$ and the distances between the VeNB and MUEs $\left(d_{1}\right.$ and $\left.d_{2}\right)$ are calculated according to the location information that MUE\#1 and MUE\#2 send back to the VeNB. Then, the distance information can be used to estimate the received power of the VeNB and MUEs for LIPA and D2D links. On the other hand, the received power of the VeNB and MUEs for LIPA and D2D links can be measured by the VeNB and MUEs. Therefore, the received power with estimation and measurement between the VeNB and MUEs (LIPA link) and between MUEs (D2D link) can be used as criteria for switching the transmission mode. If the received power of the direct communication between two relief workers (in D2D mode) is stronger than that in the LIPA mode, the D2D link is selected for communication; otherwise, the LIPA link is chosen.

In the case of using the received power as a criterion for LIPA/D2D switching, the received power of the D2D link $\left(P_{r}^{\mathrm{D} 2 \mathrm{D}}\right)$ is calculated according to the MUE transmit power and the path loss of the transmission distance. In the LIPA mode, the uplink received power $\left(P_{r}^{\mathrm{LIPA}, \mathrm{UL}}\right)$ and the downlink received power $\left(P_{r}^{\mathrm{LIAA}, \mathrm{DL}}\right)$ can be estimated based on the largescale and small-scale fading channels and the transmission power of the VeNB and MUEs. Therefore, the received power of the actual receiver in the LIPA mode $\left(P_{r}^{\mathrm{LIPA}}\right)$ is the smaller received power between the uplink and downlink. Following the calculation of the received power in the D2D and LIPA modes, a judgement is made regarding which received power is greater. If the received power in the LIPA mode is higher than that in the D2D mode, the LIPA mode is selected; otherwise, the D2D mode is selected, as shown in Algorithm 1.

In the case of using the distance as a criterion for LIPA/D2D switching, the distance in the D2D mode is the distance between MUEs $\left(d_{3}\right)$. In the LIPA mode, the uplink distance is the distance between MUE\# 1 and the $\operatorname{VeNB}\left(d_{1}\right)$, which is calculated according to the location coordinate sent by MUE\# 1 back to the VeNB. The downlink distance is the distance between VeNB and MUE\#2 $\left(d_{2}\right)$, which is calculated according to the location coordinate sent by MUE\#2 back to the VeNB. The uplink and downlink distances are calculated in parallel to obtain the reference values of the reception distance $\left(d^{*}\right)$ in the LIPA mode. After the reference values of the distances $\left(d^{*}\right)$ in the D2D and LIPA modes are calculated, a judgement is made regarding which is greater. If the distance in the LIPA mode is greater than that in the D2D mode, i.e., the distance between MUE\#1 and MUE\#2 is shorter; the D2D mode is selected; otherwise, the LIPA mode is selected, as shown in Algorithm 2.

\section{Simulation Results and Analysis}

In this paper, we use MATLAB to simulate the architecture of the disaster-resilient communication networks. The simulation scenario consists of one VeNB and two MUES. Table 3 presents the simulation parameter settings in the 


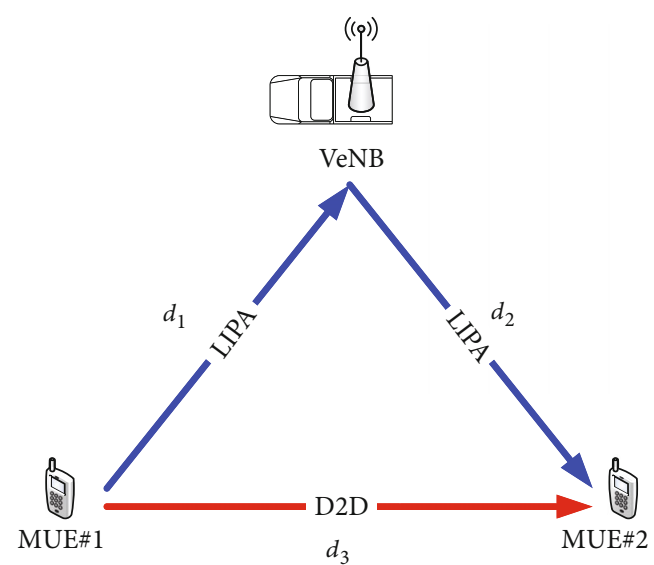

Figure 5: Schematic diagram of the traffic offloading mode switching scenario.

hybrid LIPA/D2D, LIPA Only, and D2D Only modes. The service area of the VeNB is set as 500 meters. The SAR team entered the disaster area in pairs. Therefore, there are two MUEs in the disaster area, and the initial distance between the pair of relief workers is set to 10 meters. The mobility rate ranges from $3 \mathrm{~km} / \mathrm{h}$ to $30 \mathrm{~km} / \mathrm{h}$. The transmit power of the MUEs and the VeNB is set as $23 \mathrm{dBm}$ and $36 \mathrm{dBm}$, respectively. The white Gaussian noise power is set as $-174 \mathrm{dBm} / \mathrm{Hz}$.

According to the mode switching criterion, four traffic offloading modes are simulated and analyzed within the SAR time, including (1) the hybrid mode based on the received power, (2) the hybrid mode based on the reception distance, (3) the D2D Only mode, and (4) the LIPA Only mode. The user mobility is also considered as shown in Section 2.3. According to the simulation results at different velocities and the SAR time for RWM and RDM schemes, the hybrid traffic offloading mechanism proposed in this paper can effectively improve the average effective spectrum efficiency at different velocities and a constant SAR time.

4.1. The Effect of the Mobility. Figure 6 shows the average effective spectrum efficiency against the velocity of MUEs under the RWM scheme for the four traffic offloading modes. From the figure, we have the following observations:

(1) For the D2D Only mode in RWM, the spectrum efficiency decreases as the velocity of MUEs increases. This is because the variance of the distance is smaller when the MUEs move at low speeds. As the speed increases, the variance of distance increases. The two MUEs at a low velocity can maintain a shorter distance than those at a high velocity. Consequently, the D2D Only mode with low speed has a better spectrum efficiency than that with a high speed. However, the spectrum efficiency of the LIPA Only mode keeps almost the same value, irrespective of the velocity variation of MUEs. Since the VeNB can provide the stable signal strength to MUEs with the LIPA Only mode in the coverage of disaster area, the average effective spectrum efficiency can stay a near constant. In this example, the D2D Only mode can achieve $41.58 \%$ more spectrum efficiency than the LIPA Only mode when the velocity of MUEs is at $3 \mathrm{~km} / \mathrm{h}$. However, as the velocity of MUEs increases to $30 \mathrm{~km} / \mathrm{h}$, the LIPA Only mode can achieve $196.92 \%$ more spectrum efficiency than the D2D Only mode. Therefore, when the velocity of MUEs is below $8 \mathrm{~km} / \mathrm{h}$, the D2D Only mode can provide better spectrum efficiency than the LIPA Only mode. On the other hand, the LIPA Only mode can provide better spectrum efficiency than the D2D Only mode when the velocity of MUEs is above $8 \mathrm{~km} / \mathrm{h}$.

(2) For the hybrid mode, the spectrum efficiency also decreases as the velocity of MUEs increases due to the increasing variance of distance. Nevertheless, the power criterion scheme can achieve higher spectrum efficiency than the distance criterion scheme. This is because the power criterion scheme is based on the received power measured by the VeNB and MUEs while the distance criterion scheme is based on the reception distance estimated by GPS information. The estimation of the reception distances exists some errors from the measurement of the received power because of the channel effect. However, the error between the power criterion scheme and the distance criterion scheme is insignificant, and the complexity of the power criterion scheme is more than that of the distance criterion scheme. In this example, the power criterion scheme can achieve $0.36 \%$ and $2.06 \%$ more spectrum efficiency than the distance criterion scheme when the velocity of MUEs is at $3 \mathrm{~km} / \mathrm{h}$ and $30 \mathrm{~km} / \mathrm{h}$, respectively.

(3) Both schemes of the proposed hybrid mode can achieve higher spectrum efficiency than the D2D Only mode and the LIPA Only mode at an arbitrary velocity, because the proposed hybrid LIPA/D2D traffic offloading mode can switch the appropriate traffic offloading mode based on the current communication environment. In the example, when the velocity of MUEs is $3 \mathrm{~km} / \mathrm{h}$, the power criterion scheme of the hybrid mode can achieve $43.03 \%$ and $1.03 \%$ higher spectrum efficiency than the LIPA Only mode and the D2D Only mode, respectively. Moreover, the distance criterion scheme of the hybrid mode can achieve $42.51 \%$ and $0.66 \%$ higher spectrum efficiency than the LIPA Only mode and the D2D Only mode, respectively. As the velocity of MUEs approaches $30 \mathrm{~km} / \mathrm{h}$, the power criterion scheme of the hybrid mode can achieve $6.12 \%$ and $215.10 \%$ higher spectrum efficiency than the LIPA Only mode and the D2D Only mode, respectively. Furthermore, the distance criterion scheme of the hybrid mode can achieve $3.98 \%$ and $208.75 \%$ higher spectrum efficiency than the LIPA Only mode and the D2D Only mode, respectively. 


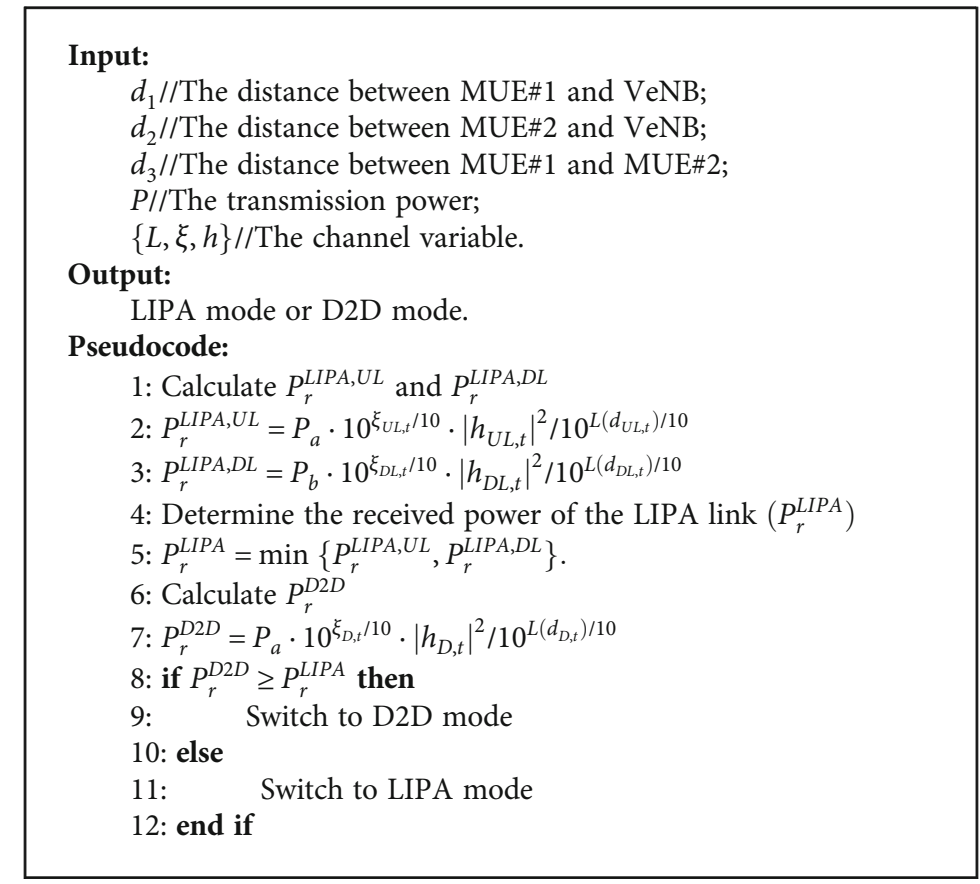

Algorithm 1: Hybrid LIPA/D2D mode selection based on received power.

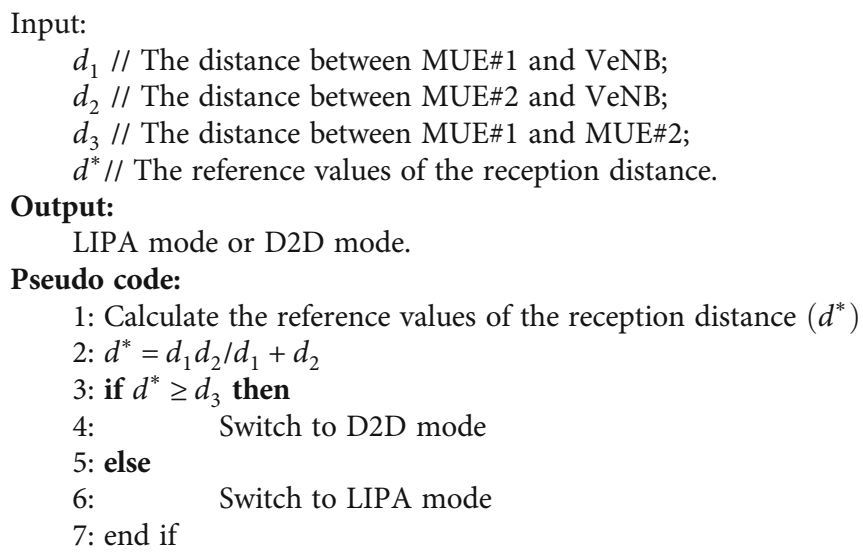

Algorithm 2: Hybrid LIPA/D2D mode selection based on reception distance.

TABLE 3: Simulation parameter settings.

\begin{tabular}{lc}
\hline Parameters & Values \\
\hline VeNB radius $\left(R_{m}\right)$ & $500 \mathrm{~m}$ \\
Initial distance between two MUEs $\left(R_{d}\right)$ & $10 \mathrm{~m}$ \\
Velocity of MUEs & $3-30 \mathrm{~km} / \mathrm{h}$ \\
Transmission power (MUE/VeNB) & $23 / 36 \mathrm{dBm}[24]$ \\
Noise power $(N 0)$ & $-174 \mathrm{dBm} / \mathrm{Hz}$ \\
Noise figure of VeNB/MUE & $7 \mathrm{~dB}$ \\
SAR time & $1-24 \mathrm{hours}$ \\
Shadowing $(\xi)$ & $10 \mathrm{~dB}$ \\
\hline
\end{tabular}

Figure 7 shows the average effective spectrum efficiency against the velocity of MUEs under the RDM scheme for the four traffic offloading modes. From the figure, we have the following observations:

(1) For the four traffic offloading modes in RDM scheme, the average effective spectrum efficiency does not vary too much when the velocity of MUEs increases. That means the average effective spectrum efficiency is irrespective of the velocity of MUEs in the RDM scheme. In this example, when the velocity of MUEs is $3 \mathrm{~km} / \mathrm{h}$, the spectrum efficiency of the power criterion scheme of the hybrid mode, the distance criterion scheme of the hybrid mode, the LIPA Only mode, and the D2D Only mode is $3.05 \mathrm{bits} / \mathrm{s} / \mathrm{Hz}, 3.01 \mathrm{bits} / \mathrm{s} / \mathrm{Hz}, 2.92$ 


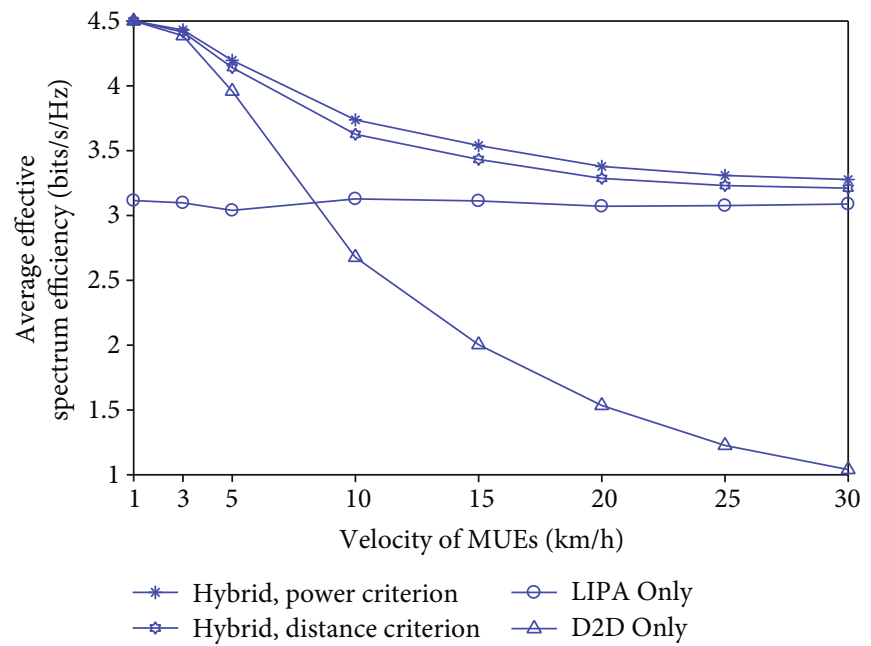

FIGURE 6: Average effective spectrum efficiency versus the velocity of MUEs under the RWM scheme.

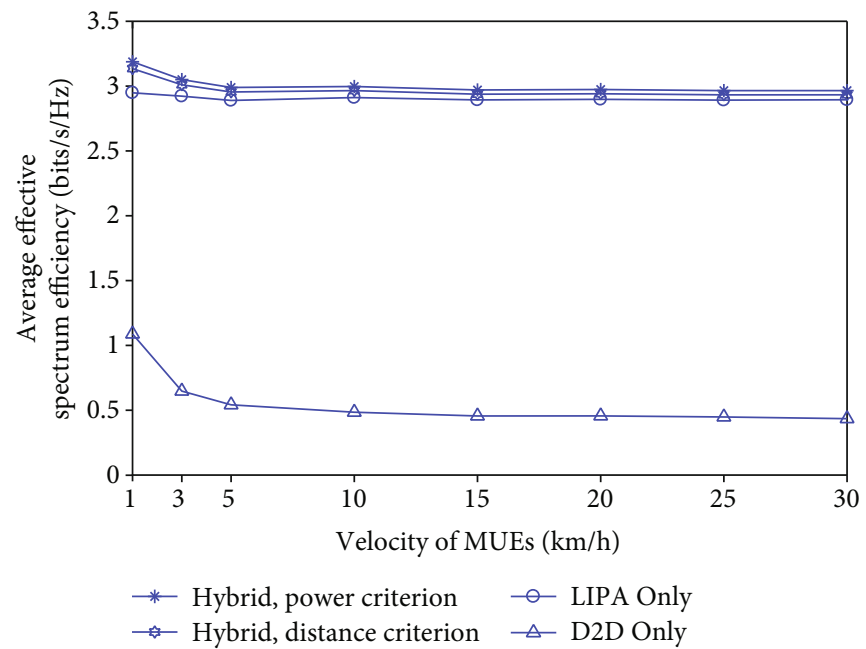

FIgURE 7: Average effective spectrum efficiency versus the velocity of MUEs under the RDM scheme.

bits $/ \mathrm{s} / \mathrm{Hz}$, and $0.65 \mathrm{bits} / \mathrm{s} / \mathrm{Hz}$, respectively. As the velocity of MUEs increases to $30 \mathrm{~km} / \mathrm{h}$, the spectrum efficiency of the power criterion scheme of the hybrid mode, the distance criterion scheme of the hybrid mode, the LIPA Only mode, and the D2D Only mode is $2.97 \mathrm{bits} / \mathrm{s} / \mathrm{Hz}, 2.93 \mathrm{bits} / \mathrm{s} / \mathrm{Hz}, 2.90 \mathrm{bits} / \mathrm{s} / \mathrm{Hz}$, and $0.43 \mathrm{bits} / \mathrm{s} / \mathrm{Hz}$, respectively. It shows that the impact of the MUE velocity is very small in RDM for the four traffic offloading modes.

(2) The power criterion scheme of the hybrid mode, the distance criterion scheme of the hybrid mode, and the LIPA Only mode have the similar average effective spectrum efficiency. However, both schemes of the hybrid mode can perform better spectrum efficiency than the LIPA Only mode. In this example, the power criterion scheme of the hybrid mode and the distance criterion scheme of the hybrid mode can achieve $4.38 \%$ and $3.01 \%$ more spectrum effi- ciency than the LIPA Only mode when the velocity of MUEs is at $3 \mathrm{~km} / \mathrm{h}$. As the velocity of MUEs increases to $30 \mathrm{~km} / \mathrm{h}$, the power criterion scheme of the hybrid mode and the distance criterion scheme of the hybrid mode can achieve $2.42 \%$ and $1.31 \%$ more spectrum efficiency than the LIPA Only mode. It is shown that the LIPA Only is applicable in the RDM scheme. Nevertheless, the hybrid mode can achieve more performance.

(3) Moreover, the power criterion scheme of the hybrid mode has the best spectrum efficiency, and the D2D Only mode has the worst performance. This is because the RDM scheme makes each MUE search in one random direction and then search in another random direction when the MUE reaches the boundary of the disaster area. Therefore, the separation distance between the two MUEs is usually too far during the rescue operation to benefit from the 
D2D Only mode. In this example, the power criterion scheme of the hybrid mode, the distance criterion scheme of the hybrid mode, and the LIPA Only mode can achieve 371.04\%, 364.86\%, and $351.27 \%$ more spectrum efficiency than the D2D Only mode when the velocity of MUEs is at $3 \mathrm{~km} / \mathrm{h}$. As the velocity of MUEs increases to $30 \mathrm{~km} / \mathrm{h}$, the power criterion scheme of the hybrid mode, the distance criterion scheme of the hybrid mode, and the LIPA Only mode can achieve $581.70 \%, 574.34 \%$, and $565.61 \%$ more spectrum efficiency than the D2D Only mode. The result shows that the D2D Only mode is not applicable in the RDM scheme.

4.2. The Effect of the SAR Time. Figure 8 shows the average effective spectrum efficiency against the SAR time of MUEs under the RWM scheme for the four traffic offloading modes. From the figure, we have the following observations:

(1) For the D2D Only mode in RWM, the spectrum efficiency decreases as the SAR time of MUEs increases. This is because the variance of the distance between MUEs is small in the beginning of the rescue operation for RWM scheme. As the SAR time increases, the region of the rescue becomes more and more broad and so is the distance of MUEs. Accordingly, the D2D Only mode cannot effectively achieve a better spectrum efficiency with a long SAR time. On the other hand, the LIPA Only mode has a nearly constant spectrum efficiency no matter how long the SAR time is. In this example, the D2D Only mode can achieve $25.39 \%$ more spectrum efficiency than the LIPA Only mode when the SAR time of MUEs is three hours. However, as the SAR time of MUEs increases to 24 hours, the LIPA Only mode can achieve $53.85 \%$ more spectrum efficiency than the D2D Only mode. Therefore, if the SAR time of MUEs is below eight hours, the D2D Only mode can provide better spectrum efficiency than the LIPA Only mode. However, the LIPA Only mode can provide better spectrum efficiency than the D2D Only mode when the SAR time of MUEs is above eight hours.

(2) Similar to the D2D Only mode, both of the hybrid mode have the decreasing spectrum efficiency when the SAR time of MUEs is increasing. Although the power criterion scheme can achieve more spectrum efficiency than the distance criterion scheme, the distance criterion scheme has less complexity than the power criterion scheme. Furthermore, the difference in spectrum efficiency between the measurement of the received power and the estimation of the reception distances due to the channel effect is slight. In this example, the power criterion scheme can achieve $1.56 \%$ and $3.08 \%$ more spectrum efficiency than the distance criterion scheme when the SAR time of MUEs is 3 hours and 24 hours, respectively.
(3) Since the proposed hybrid LIPA/D2D traffic offloading mode can switch the proper traffic offloading mode according to the real communication environment, both schemes of the hybrid mode can achieve higher spectrum efficiency than the D2D Only mode and the LIPA Only mode with a given SAR time. In the example, when the SAR time of MUEs is three hours, the power criterion scheme of the hybrid mode can achieve $35.13 \%$ and $7.77 \%$ higher spectrum efficiency than the LIPA Only mode and the D2D Only mode, respectively. In addition, the distance criterion scheme of the hybrid mode can achieve $33.05 \%$ and $6.11 \%$ higher spectrum efficiency than the LIPA Only mode and the D2D Only mode, respectively. If the SAR time of MUEs is 24 hours, the power criterion scheme of the hybrid mode can achieve $14.18 \%$ and 75.77\% higher spectrum efficiency than the LIPA Only mode and the D2D Only mode, respectively. Moreover, the distance criterion scheme of the hybrid mode can achieve $10.77 \%$ and $70.53 \%$ higher spectrum efficiency than the LIPA Only mode and the D2D Only mode, respectively.

Figure 9 shows the average effective spectrum efficiency against the SAR time of MUEs under the RDM scheme for the four traffic offloading modes. From the figure, we have the following observations:

(1) In light of the figure, the influence of the SAR time on the average effective spectrum efficiency is insignificant for the four traffic offloading modes in RDM scheme. In this example, when the SAR time of MUEs is three hours, the spectrum efficiency of the power criterion scheme of the hybrid mode, the distance criterion scheme of the hybrid mode, the LIPA Only mode, and the D2D Only mode is 2.98 bits/s/Hz, $2.95 \mathrm{bits} / \mathrm{s} / \mathrm{Hz}, 2.90 \mathrm{bits} / \mathrm{s} / \mathrm{Hz}$, and 0.49 bits/s/Hz, respectively. As the SAR time of MUEs increases to 24 hours, the spectrum efficiency of the power criterion scheme of the hybrid mode, the distance criterion scheme of the hybrid mode, the LIPA Only mode, and the D2D Only mode is 2.96 bits/s/Hz, $2.93 \mathrm{bits} / \mathrm{s} / \mathrm{Hz}, 2.89 \mathrm{bits} / \mathrm{s} / \mathrm{Hz}$, and 0.43 bits/s/Hz, respectively.

(2) Both schemes of the hybrid mode and the LIPA Only mode have the similar average effective spectrum efficiency. Nevertheless, the power criterion scheme of the hybrid mode has the best spectrum efficiency, and the distance criterion scheme of the hybrid mode has better spectrum efficiency than the LIPA Only mode. In this example, the power criterion scheme of the hybrid mode and the distance criterion scheme of the hybrid mode can achieve $2.97 \%$ and $1.80 \%$ more spectrum efficiency than the LIPA Only mode when the SAR time of MUEs is three hours. As the SAR time of MUEs increases to 24 hours, the power criterion scheme of the hybrid mode and the distance criterion scheme of the hybrid mode can achieve $2.35 \%$ and 


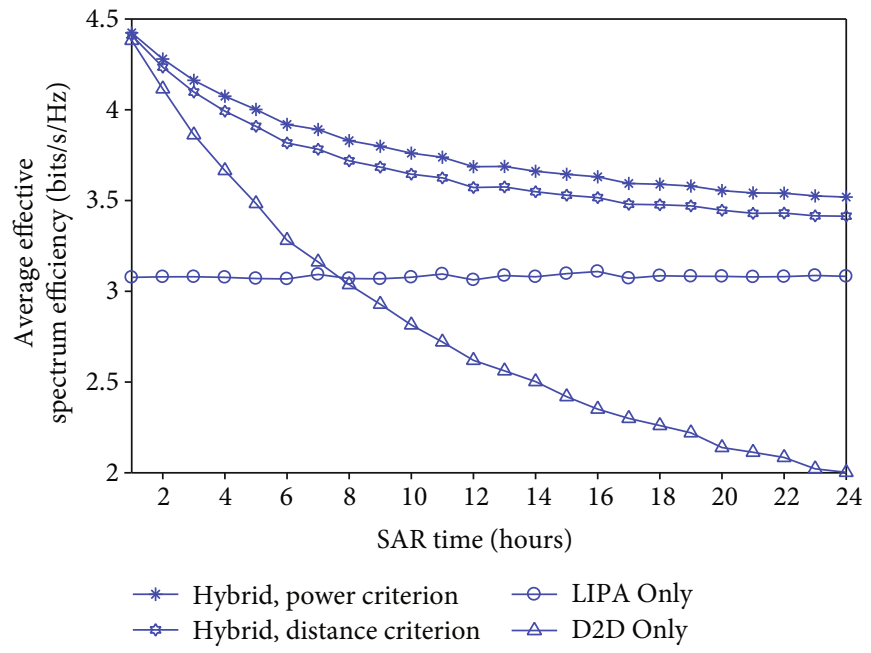

FIGURE 8: Average effective spectrum efficiency versus the SAR time of MUEs under the RWM scheme.

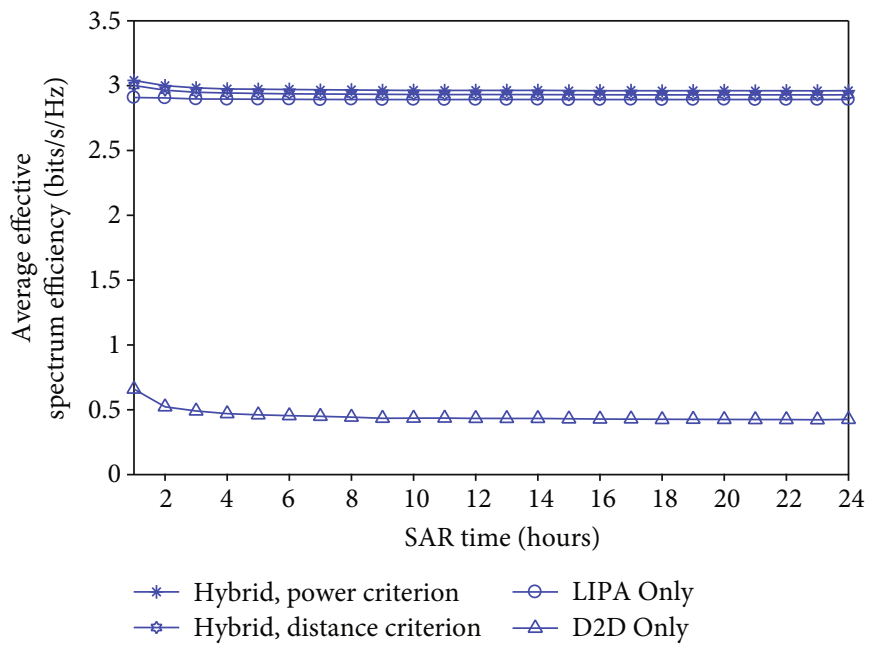

FIGURE 9: Average effective spectrum efficiency versus the SAR time of MUEs under the RDM scheme.

$1.24 \%$ more spectrum efficiency than the LIPA Only mode. Although the LIPA Only is applicable in the RDM scheme, the hybrid LIPA/D2D traffic offloading mode can achieve more performance.

(3) The power criterion scheme of the hybrid mode has the best spectrum efficiency, while the D2D Only mode has the worst performance. Because the distance between the two MUEs during the rescue operation in RDM scheme cannot be kept within an acceptable range, the D2D Only mode cannot provide the satisfactory quality of service for the disaster-resilient communication network. In this example, the power criterion scheme of the hybrid mode, the distance criterion scheme of the hybrid mode, and the LIPA Only mode can achieve $508.03 \%, 501.10 \%$, and $490.50 \%$ more spectrum efficiency than the D2D Only mode when the SAR time of MUEs is 3 hours. As the SAR time of MUEs increases to 24 hours, the power criterion scheme of the hybrid mode, the distance criterion scheme of the hybrid mode, and the LIPA Only mode can achieve $503.55 \%, 497.02 \%$, and $489.69 \%$ more spectrum efficiency than the D2D Only mode. It is shown that the LIPA Only mode is more applicable than the D2D Only mode in the RDM scheme, and the hybrid LIPA/D2D traffic offloading mode can improve more spectrum efficiency.

4.3. Summary. The RWM scheme can simulate a scenario of user mobility in which the SAR of relief workers is narrowed or expanded within a fixed area, while the RDM scheme can simulate the linear movement of relief workers (fixed-point movement or casualty transport). The distance between relief workers changes with respect to the velocity or SAR time. In the D2D Only mode, the average effective spectrum efficiency depends on the reception distance or received 
power between relief workers. In the LIPA Only mode, messages are forwarded by the VeNB to the receiver, therefore the average effective spectrum efficiency is less likely to be affected by the reception distance or received power. Since the initial locations of relief workers are adjacent, the average effective spectrum efficiency in the D2D Only mode is higher than that in the LIPA Only mode. However, the distance between relief workers increases with the user mobility and SAR time, and the average effective spectrum efficiency in the LIPA mode may be higher than that in the D2D mode. In addition, the spectrum efficiency of the RDM scheme is lower than that of the RWM scheme for the D2D Only mode.

According to the characteristics of the two aforementioned mobility models and the mode switching standards, the hybrid traffic offloading mode can select a better mode (D2D or LIPA) for obtaining the highest average effective spectrum efficiency. That is, when the distance between relief workers is short enough to provide adequate signal quality with velocity or SAR time, the average effective spectrum efficiency of the hybrid mode can exhibits a similar trend to that of the D2D Only mode. However, the average effective spectrum efficiency of the hybrid mode can exhibit a similar trend to that of the LIPA Only mode if the distance between relief workers is too long to provide adequate signal quality with velocity or SAR time. Therefore, the average effective spectrum efficiency in the hybrid mode changes depending on whether LIPA mode or D2D mode is selected. In addition, when the received power is used as the criterion for mode switching, the average effective spectrum efficiency is slightly higher than that when the distance is used as the criterion for mode switching. However, the relative complexity is higher. Therefore, by sacrificing a little performance, the distance is a good choice as the criterion for mode switching.

\section{Conclusions}

This paper investigated the effect of user mobility on the spectrum efficiency with four traffic offloading modes for disaster-resilient communication networks. The LIPA Only mode needs the eNBs surviving in the disaster area or the VeNB entering the disaster area to provide communication services for relief workers in the disaster area. If eNBs in the disaster area are destroyed or the VeNB cannot enter the disaster area owing to terrain constraints, the LIPA link is interrupted. In the D2D Only mode, because the transmit power of communication devices such as mobile phones is small, the communication quality deteriorates owing to the complex terrain or distance between relief workers in the disaster area. The hybrid LIPA/D2D traffic offloading mode can overcome the limitations of both LIPA Only mode and D2D Only mode to provide a more stable emergency communication system. This is because the proposed hybrid LIPA/D2D traffic offloading mechanism can switch LIPA link or D2D link based on the criterion of the received power or reception distance between MUEs of relief workers and the VeNB. In addition, the overall average effective spectrum efficiency is evaluated according to the different mobility characteristics (i.e., RWM and RDM) of the relief workers. When the hybrid LIPA/D2D traffic offloading mechanism is used, the local communication traffic generated during the rescue operation is not sent back to the core network, reducing the load of the core network. In this manner, the quality of communication from the disaster area to the outside network is ensured, which is beneficial to the implementation of disaster relief.

\section{Data Availability}

No datasets were generated or analyzed during the current study.

\section{Conflicts of Interest}

The authors declare that they have no conflicts of interest.

\section{Acknowledgments}

This work was sponsored by the Ministry of Science and Technology (MOST) of Taiwan under grants MOST 1092221-E-606-007-, MOST 110-2634-F-009-021-, and MOST 1102221-E-A49-039-MY3. The authors would like to thank Wen-Pin Chen for his assistance in simulating traffic offloading modes in the experiment.

\section{References}

[1] 3GPP," "Technical Specifications and Technical Reports for a UTRAN-based 3GPP system," 3GPP TSG SSA, Tech. Rep. TS 21.101 V14.1.0, 2017.

[2] Y. Shibata, N. Uchida, and N. Shiratori, "Analysis of and proposal for a disaster information network from experience of the Great East Japan Earthquake," IEEE Communications Magazine, vol. 52, no. 3, pp. 44-50, 2014.

[3] R. Maallawi, N. Agoulmine, B. Radier, and T. B. Meriem, "A comprehensive survey on offload techniques and management in wireless access and core networks," IEEE Communications Surveys \& Tutorials, vol. 17, no. 3, pp. 1582-1604, 2015.

[4] M. J. Yang, S. Y. Lim, H. J. Park, and N. H. Park, "Solving the data overload: device-to-device bearer control architecture for cellular data offloading," IEEE Vehicular Technology Magazine, vol. 8, no. 1, pp. 31-39, 2013.

[5] 3GPP, "Report on the Design and Evaluation of the Tuak Algorithm Set: A Second Example Algorithm Set for the 3GPP Authentication and Key Generation Functions," 3GPP TSG SSA, Tech. Rep. TS 35.934 V14.0.0, 2017.

[6] D. Jalihal, R. D. Koilpillai, P. Khawas et al., "A rapidly deployable disaster communications system for developing countries," in 2012 IEEE International Conference on Communications (ICC), pp. 6339-6343, Ottawa, ON, Canada, June 2012.

[7] S. Y. Lien, C. C. Chien, F. M. Tseng, and T. C. Ho, "3GPP device-to-device communications for beyond $4 \mathrm{G}$ cellular networks," IEEE Communications Magazine, vol. 54, no. 3, pp. 28-35, 2016.

[8] K. Doppler, M. Rinne, C. Wijting, C. B. Ribeiro, and K. Hugl, "Device-to-device communication as an underlay to LTEadvanced networks," IEEE Communications Magazine, vol. 7, no. 12, pp. 42-49, 2009. 
[9] K. Doppler, C.-H. Yu, C. B. Ribeiro, and P. Jänis, "Mode selection for device-to-device communication underlaying an LTEadvanced network," in 2010 IEEE Wireless Communication and Networking Conference, pp. 1-6, Sydney, NSW, Australia, April 2010.

[10] C. H. Chang, H. L. Chao, C. L. Liu, and K. L. Huang, "A local IP access mechanism for VoIP service in LTE home eNodeB systems," in 2013 Seventh International Conference on Innovative Mobile and Internet Services in Ubiquitous Computing, pp. 1-6, Taichung, Taiwan, July 2013.

[11] E. Frlan, Direct communication wireless radio system, vol. 6047178, 2000United States Patent, US, 2000.

[12] M. F. Khan, M. I. Khan, and K. Raahemifar, "Local IP Access (LIPA) enabled 3G and 4G femtocell architectures," in 2011 24th Canadian Conference on Electrical and Computer Engineering(CCECE), pp. 001049-001053, Niagara Falls, ON, Canada, May 2011.

[13] C. B. Sankaran, "Data offloading techniques in 3GPP Rel-10 networks: a tutorial," IEEE Communications Magazine, vol. 50, no. 6, pp. 46-53, 2012.

[14] H. Tang and Z. Ding, "Mixed mode transmission and resource allocation for D2D communication," IEEE Transactions on Wireless Communications, vol. 15, no. 1, pp. 162-175, 2016.

[15] C.-C. Lai and C.-M. Liu, "A mobility-aware approach for distributed data update on unstructured mobile P2P networks," Journal of Parallel and Distributed Computing, vol. 123, pp. 168-179, 2019.

[16] C.-M. Liu and C.-C. Lai, "A group-based data-driven approach for data synchronization in unstructured mobile P2P systems," Wireless Networks, vol. 27, no. 7, pp. 2465-2482, 2018.

[17] A. Orsino, A. Ometov, G. Fodor et al., "Effects of heterogeneous mobility on D2D- and drone-assisted mission-critical MTC in 5G," IEEE Communications Magazine, vol. 55, no. 2, pp. 79-87, 2017.

[18] X. Xu, Y. Zhang, Z. Sun, Y. Hong, and X. Tao, “Analytical modeling of mode selection for moving D2D-enabled cellular networks," IEEE Communications Letters, vol. 20, no. 6, pp. 1203-1206, 2016.

[19] 3GPP, "Selection Procedures for the Choice of Radio Transmission Technologies of the UMTS," 3GPP, Tech. Rep. TR 30.03U V3.2.0, 1998.

[20] 3GPP, "Radio Frequency (RF) Requirements for LTE Pico Node B," 3GPP TSG RAN, Tech. Rep. TR 36.931 V10.0.0, 2011.

[21] 3GPP, "Study on LTE Device to Device Proximity Services," 3GPP TSG RAN, Tech. Rep. TR 36.843 V12.0.1, 2014.

[22] 3GPP, "Study on Channel Model for Frequencies from 0.5 to 100 GHz," 3GPP TSG RAN, Tech. Rep. TR 38.901 V16.1.0, 2019.

[23] B. V. R. Gorantla and N. B. Mehta, "Resource and computationally efficient subchannel allocation for D2D in multicell scenarios with partial and asymmetric CSI," IEEE Transactions on Wireless Communications, vol. 18, no. 12, pp. 5806-5817, 2019.

[24] W.-K. Lai, Y.-C. Wang, H.-C. Lin, and J.-W. Li, "Efficient resource allocation and power control for LTE-A D2D communication with pure D2D model," IEEE Transactions on Vehicular Technology, vol. 69, no. 3, pp. 3202-3216, 2020. 